10,19

\title{
Колебательные и тепловые свойства оксианионных кристаллов
}

\author{
(ㄷ Д.В. Корабельников
}

Кемеровский государственный университет, Кемерово, Россия

E-mail: dkorabelnikov@yandex.ru

(Поступила в Редакцию 3 июля 2017 г.

В окончательной редакции 22 июля 2017 г.)

Колебательные и тепловые свойства доломита, хлоратов и перхлоратов щелочных металлов изучены в градиентном приближении теории функционала плотности, методом линейной комбинации атомных орбиталей. Вычислены частоты длинноволновых колебаний, ИК и КР-спектры, модовые параметры Грюнайзена. Также вычислены параметры уравнения состояния, термодинамические потенциалы, энтропия, теплоемкость, коэффициент теплового расширения. Установлено, что коэффициент теплового расширения для доломита значительно меньше, чем для хлоратов и перхлоратов. Показано, что при $T>200 \mathrm{~K}$ температурная зависимость теплоемкости определяется преимущественно внутримолекулярными колебаниями.

Работа выполнена при поддержке Министерства образования и науки РФ в рамках контракта № $15.3487 .2017 / П Ч$.

DOI: 10.21883/FTT.2018.03.45563.216

\section{1. Введение}

Оксианионные кристаллы - соединения с молекулярным кислородсодержащим анионом, обладающие разнообразными свойствами и имеющие широкое практическое применение. Карбонаты являются основными участниками углеродного цикла в мантии Земли $[1,2]$. $\mathrm{B}$ частности $\mathrm{MgCa}\left(\mathrm{CO}_{3}\right)_{2}$ (доломит) представляет собой одну из фаз, в которой углерод содержится в мантии и является минеральным компонентом углей [3,4]. Доломит используется при производстве цемента и стекол, огнеупорных материалов, строительных материалов, для контроля загрязнения промышленных объектов, использовался при ликвидации аварии на Чернобыльской АЭС. Его также используют в качестве добавки в фармацевтической промышленности [5].

Хлораты и перхлораты щелочных металлов используются в качестве энергетических окислителей в твердых ракетных топливах, во взрывчатых и пиротехнических системах, а также в качестве источников кислорода в дыхательных аппаратах [6-8]. Перхлорат лития является одним из наиболее перспективных кандидатов для окислителей в композитных ракетных топливах из-за низкого атомного веса лития и высокого содержания кислорода [8]. Также перхлорат лития применяется как компонент электролитов для твердотельных литиевых батарей [9]. Интересно отметить, что сравнительно недавно перхлораты были обнаружены на Марсе [10] и могут быть полезны для его будущих исследований.

Доломит $\mathrm{CaMg}\left(\mathrm{CO}_{3}\right)_{2}$ имеет гексагональную структуру с одной формульной единицей $(Z)$ в элементарной ячейке [11]. Хлорат натрия и калия кристаллизуются в кубическую и моноклинную структуры соответственно $[12,13]$, тогда как перхлораты щелочных металлов имеют ромбическую структуру [14-16]. Элементарная ячейка хлората натрия, перхлоратов лития и калия содержит четыре формульные единицы. Для хлората калия и перхлората натрия $Z=2$.

Колебательные и тепловые свойства доломита, хлоратов и перхлоратов щелочных металлов экспериментально изучались в работах [17-30]. Исследование внутримолекулярных и решеточных колебаний $\mathrm{CaMg}\left(\mathrm{CO}_{3}\right)_{2}$ методом рамановской (КР) спектроскопии проведено в [17-19]. Колебательные моды хлората натрия методом ИК-спектроскопии изучены в [20]. В работах [21-24] КР-спектры частот измерены для хлората калия и перхлоратов $\mathrm{NaClO}_{4}, \mathrm{KClO}_{4}$. Результаты экспериментальных измерений теплоемкости доломита, хлората натрия, хлората и перхлората калия представлены в [25-28]. Тепловое расширение доломита, хлоратов натрия и калия, а также перхлората калия экспериментально исследовано в [29-32]. Установлено, что с ростом температуры коэффициент теплового расширения $\mathrm{KClO}_{3}$, в отличие от доломита и хлората натрия, уменьшается.

Колебательные спектры доломита и хлоратов щелочных металлов из первых принципов изучены в $[33,34]$, однако параметры Грюнайзена, характеризующие ангармонизм колебаний и их барическую зависимость, вычислены не были. Колебательные свойства перхлоратов из первых принципов не исследованы. Электронные, оптические, поверхностные и упругие свойства хлоратов и перхлоратов щелочных металлов из первых принципов изучены в [35-38], тогда как расчеты термодинамических функций отсутствуют. Также на основе первопринципных вычислений частот колебаний не изучены тепловые свойства доломита.

В настоящей работе проведено теоретическое исследование колебательных и тепловых свойств оксианионных кристаллов (доломита, хлоратов и перхлоратов щелочных металлов), которые проанализированы с уче- 
том природы колебаний и сопоставлены с имеющимися экспериментальными данными.

\section{2. Метод расчета}

В настоящей работе расчеты статической энергии, атомной структуры и частот нормальных длинноволновых колебаний $(k=0)$ выполнены на основе теории функционала электронной плотности (DFT) с помощью программы CRYSTAL [39]. В DFT-вычислениях использовались базисные наборы линейных комбинаций атомных орбиталей $[33,40-45]$ и градиентное приближение к обмену и корреляции [46].

Первопринципное определение кристаллической структуры проводилось с помощью минимизации полной энергии, межатомных сил и смещений по известной схеме BFGS [47]. Гармонические частоты фононов в точке $Г$ получаются из диагонализации масс-взвешенной матрицы Гессе вторых производных энергии по атомным смещениям $u$ [48]

$$
W_{a i, b j}^{\Gamma}=\frac{H_{a i, b j}^{0}}{\sqrt{M_{a} M_{b}}}, \quad H_{a i, b j}^{0}=\left(\frac{\partial^{2} E}{\partial u_{a i}^{0} \partial u_{b j}^{0}}\right),
$$

где атомы $a$ и $b$ с атомными массами $M_{a}$ и $M_{b}$ смещаются в элементарной ячейке (индекс 0), вдоль $i$-го и $j$-го декартовых направлений соответственно. Интенсивность КР-линии Стокса фононной моды $Q_{p}$, активной вследствие $\alpha_{i i}$ компоненты тензора поляризуемости, пропорциональна

$$
I_{i i^{\prime}}^{p} \propto\left(\frac{\partial \alpha_{i i^{\prime}}}{\partial Q_{P}}\right)^{2}
$$

Относительные интенсивности пиков комбинационного рассеяния вычисляются аналитически путем использования схемы, которая представляет собой расширение аналитического расчета ИК-интенсивности [49,50]. Интенсивность для ИК-поглощения вычисляется с помощью тензора эффективного заряда Борна $Z_{i j, a}^{v}$, определяемого соотношением между поляризацией $P$ и смещением атома $u$, или между силой $F$, действующей на атом $a$ и электрическим полем $E$

$$
Z_{i j, a}^{v}=V_{0} \frac{\partial P_{i a}^{v}}{\partial u_{j a}}=\frac{\partial F_{i a}^{v}}{\partial E_{j}},
$$

где $v$ - номер колебания.

Зная каноническое уравнение состояния $F(V, T)$ однородной системы при условиях термодинамического равновесия, можно определить ее частные производные, которые позволяют получить другие термодинамические величины. Дифференцирование свободной энергии по объему определяет термическое уравнение состояния

$$
P(V, T)=-\frac{\partial F(V, T)}{\partial V} .
$$

Решением (4) является физически эквивалентное ему термическое уравнение состояния $V(P, T)$, позволяющее перейти от переменных $V, T$ к переменным $P, T$.
Дифференцирование свободной энергии по температуре определяет энтропию

$$
S(V(P, T), T)=-\frac{\partial F(V, T)}{\partial T} .
$$

Связь энергии системы $E(V, T)$ со свободной энергией и энтропией определяется выражением

$$
E(V(P, T), T)=F(V, T)+T S(V, T),
$$

дифференцированием которой можно получить теплоемкость при постоянном объеме

$$
C_{V}(V(P, T), T)=\frac{\partial E(V, T)}{\partial T} .
$$

На основе термического уравнения состояния можно получить изотермический объемный модуль $B$ и параметр Грюнайзена $\gamma$

$$
\begin{aligned}
& B(V(P, T), T)=-V \frac{\partial P(V, T)}{\partial V}, \\
& \gamma(V(P, T), T)=\frac{V}{C_{V}} \frac{\partial P(V, T)}{\partial T} .
\end{aligned}
$$

Тогда коэффициент объемного расширения можно посчитать следующим образом:

$$
\alpha(V(P, T), T)=-\frac{1}{V} \frac{\partial V(P, T)}{\partial T}=\frac{\gamma(V, T) C_{V}(V, T)}{V B(V, T)} .
$$

Коэффициент теплового расширения связан с теплоемкостью при постоянном давлении

$$
C_{p}(V(P, T), T)=C_{V}(V, T)(1+\alpha(V, T) \gamma(V, T) T) .
$$

Существует ряд общих выражений для определения $F(V, T)$ и $P(V, T)$ посредством равновесных значений $(P=0)$ для энергии, объема, упругого модуля и его производной.

Хорошо известное уравнение Виньета [51] записывается следующим образом:

$$
P(V, T)=3 B_{0} \frac{1-x}{x^{2}} \exp \left\{\frac{3}{2}\left(B_{1}-1\right)(1-x)\right\} .
$$

Здесь $x=\left(V / V_{0}\right)^{1 / 3}$. В равновесии $V_{0}=V, B_{0}=B$, $B_{1}=\partial B / \partial P$. Оно является универсальным, поскольку выполняется для большого класса соединений.

Наиболее известны модели твердого тела, основанные на квазигармоническом приближении [52]. Смысл квазигармонического приближения состоит в том, что изменение объема тела приводит лишь к изменению спектра колебаний, тогда как сами колебания остаются гармоническими. Свободная энергия в этом приближении определяется следующим образом:

$$
\begin{aligned}
& F(V, T)=E_{\text {stat }}(V) \\
& +\int_{0}^{\infty}\left(\frac{\hbar \omega}{2}+k_{\mathrm{B}} T \ln \left(1-\exp \left(-\frac{\hbar \omega}{k_{\mathrm{B}} T}\right)\right)\right) g(\omega, V) d \omega,
\end{aligned}
$$

где $E_{\text {stat }}(V)$ - энергия статической решетки, рассчитываемая стандартными методами теории функционала 

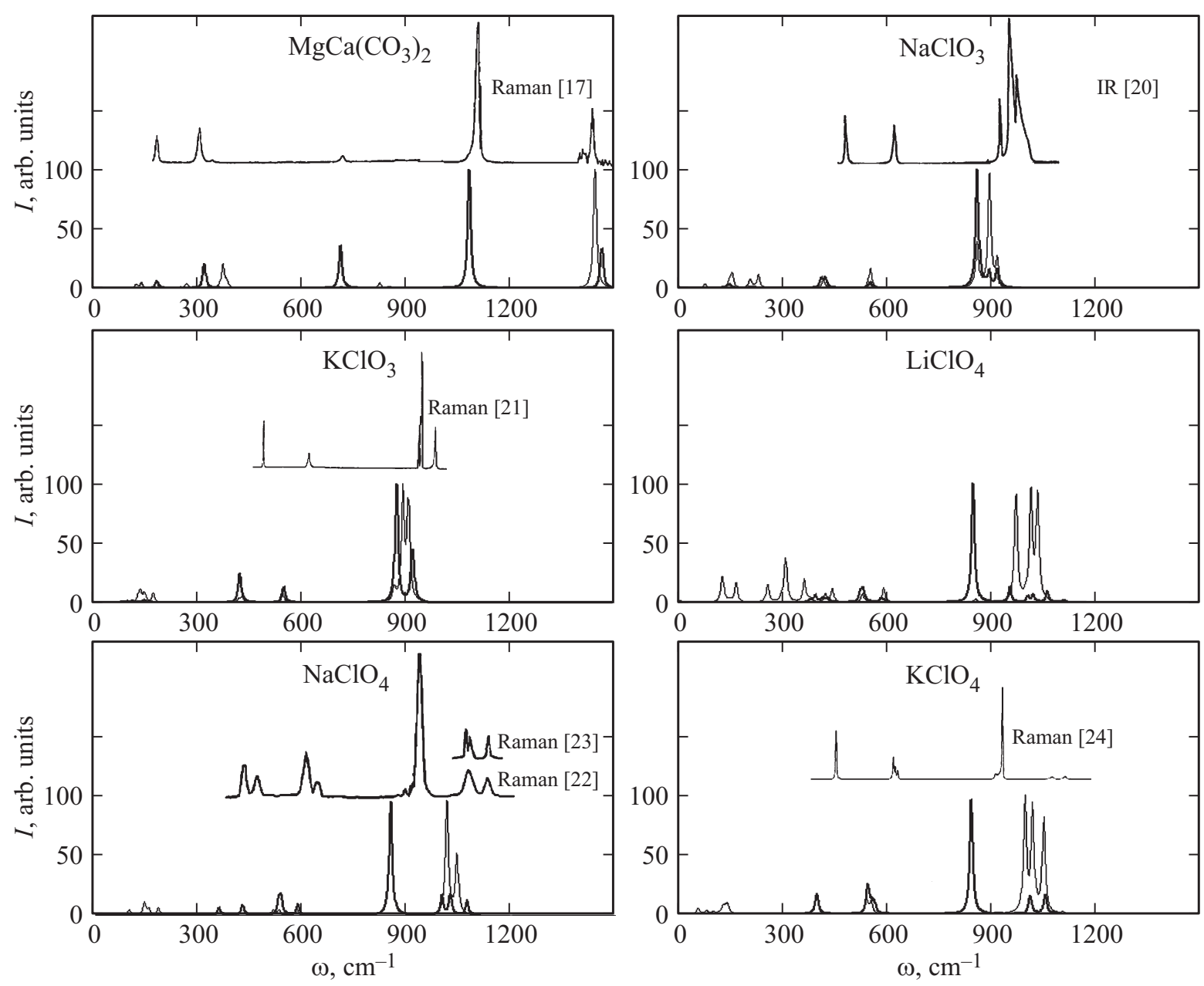

Рис. 1. Вычисленные ИК-спектры (тонкие линии), рамановские спектры (жирные линии) оксианионных кристаллов и экспериментальные спектры [17,20-24].

плотности, $\hbar-$ приведенная постоянная Планка, $k_{\mathrm{B}}-$ постоянная Больцмана. Второе слагаемое в (13) соответствует тепловой части свободной энергии $F_{T}(V, T)$. Основная задача при этом состоит в определении конкретного распределения частот $g(\omega, V)$ в спектре колебаний. Реальный вид этого распределения достаточно сложен, поэтому часто используются модельные представления. В настоящей работе использовалось приближение Дебая-Эйнштейна [53], в котором акустические моды описываются в рамках приближения Дебая, тогда как оптические - в приближении Эйнштейна. В модели Дебая-Эйнштейна используется не температура Дебая $\Theta_{D}$, а температура Дебая-Эйнштейна $\Theta_{D E}$.

Температура Дебая может быть вычислена через модуль $B$ и коэффициент Пуассона $\mu$ [54]

$$
\begin{gathered}
\Theta_{D}=\frac{\hbar}{k_{\mathrm{B}}}\left(6 \pi^{2} V^{1 / 2} N_{a}\right)^{1 / 3} f(\mu) \sqrt{\frac{B}{M}}, \\
f(\mu)=\left\{3\left[2\left(\frac{2}{3} \frac{1+\mu}{1-2 \mu}\right)^{3 / 2}+\left(\frac{1}{3} \frac{1+\mu}{1-\mu}\right)^{3 / 2}\right]^{-1}\right\}^{1 / 3} .
\end{gathered}
$$

Здесь $N_{a}-$ число атомов в формульной единице, $M$ - молярная масса. Температура Дебая-Эйнштейна определяется как

$$
\Theta_{D E}=\Theta_{D} /\left(N_{a}\right)^{1 / 3}
$$

\section{3. Колебательные свойства}

На рис. 1 представлены вычисленные ИК- и рамановские (КР) спектры доломита, хлоратов и перхлоратов щелочных металлов в сравнении с экспериментальными спектрами [17,20-24]. Видно, что структура теоретических и экспериментальных спектров в целом согласуется, а соответствующие значения частот отличаются менее чем на $10 \%$.

В вычисленном рамановском спектре доломита выделяются три полосы при 715 (723 [33]), 1087 (1101), $1470(1438) \mathrm{cm}^{-1}$, отвечающие внутримолекулярным колебаниям, и две полосы при 185 (177), $322(296) \mathrm{cm}^{-1}$ в области решеточных колебаний (рис. 1). В скобках для сравнения приведены рассчитанные значения [33]. Пик при $\sim 1100 \mathrm{~cm}^{-1}$ является самым интенсивным, 

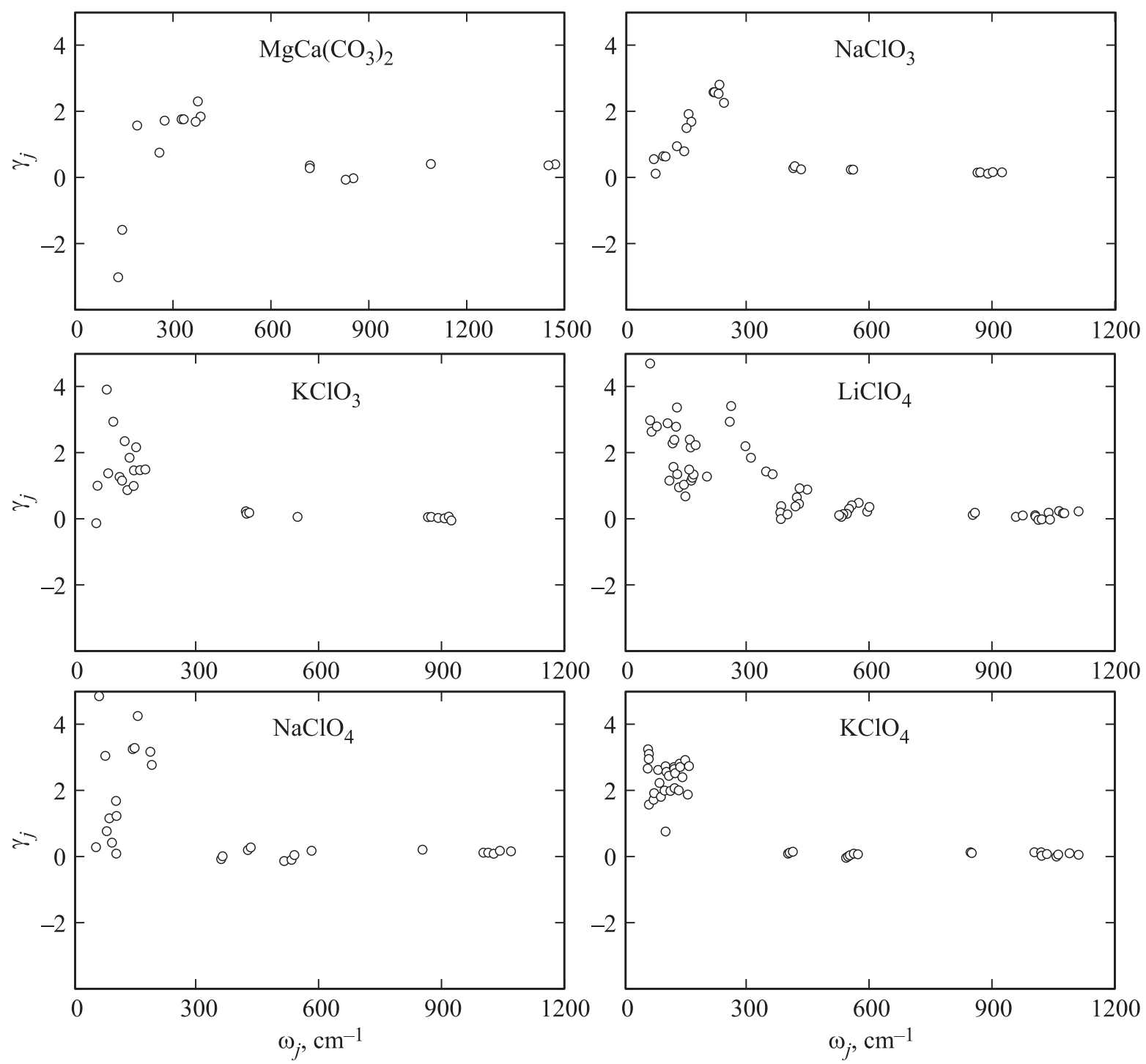

Рис. 2. Рассчитанные зависимости параметров Грюнайзена $\gamma_{j}$ от частоты $\omega_{j}$ для решеточных и внутримолекулярных мод оксианионных кристаллов.

что согласуется с экспериментом [17]. В вычисленном ИК-спектре доломита самой активной является мода с частотой $1448 \mathrm{~cm}^{-1}$. Максимумы ИК-спектра в области решеточных колебаний реализуются при 125,140 , $270,375 \mathrm{~cm}^{-1}$.

Внутримолекулярные моды, активные в ИК- и рамановских спектрах хлоратов и перхлоратов, имеют меньшие частоты. Так, частоты внутримолекулярных колебательных мод, активных в КР-спектре, для хлората натрия составили 412, 423, 553, 860, 895, $918 \mathrm{~cm}^{-1}$. При этом полоса при $\sim 900 \mathrm{~cm}^{-1}$ наиболее интенсивна, также как в теоретическом и экспериментальном рамановском спектрах хлората калия. Однако, если для $\mathrm{NaClO}_{3}$ в этой полосе выделяются три максимума при 860, 895, $918 \mathrm{~cm}^{-1}$, то для $\mathrm{KClO}_{3}$ только два при $874,921 \mathrm{~cm}^{-1}$. Для ИК-спектра хлората натрия в области высокочастотных внутримолекулярных колебаний выделяется интенсивная полоса с тремя максимумами, что согласуется с экспериментом [20]. Для ИК-спектра $\mathrm{KClO}_{3}$ наиболее активными являются три моды внутримолекулярных колебаний с частотами 867, 892, $907 \mathrm{~cm}^{-1}$. Что касается решеточных колебаний, то наиболее активными в КР-спектре $\mathrm{NaClO}_{3}$ являются моды с частотами 146, $207 \mathrm{~cm}^{-1}$, тогда как в ИК-спектре наиболее активны моды с частотами $153,206,230 \mathrm{~cm}^{-1}$. Для $\mathrm{KClO}_{3}$ наиболее активны КР-моды с частотами 94, 109, $144 \mathrm{~cm}^{-1}$ и ИКмоды с частотами $133,145,171 \mathrm{~cm}^{-1}$.

Если в КР-спектрах доломита и хлоратов выделяется три области активных внутримолекулярных колебаний, то в КР-спектрах перхлоратов их четыре $(\sim 400,550$, $\left.850,1000 \mathrm{~cm}^{-1}\right)$ и пики при $\sim 850 \mathrm{~cm}^{-1}$ самые интенсивные. При этом для $\mathrm{KClO}_{4}$ эти области более узкие, чем для перхлоратов лития и натрия, что согласуется с экспериментом [22-24]. Для $\mathrm{LiClO}_{4}\left(\mathrm{NaClO}_{4}\right)$ помимо основного максимума наблюдается сравнительно большое число менее интенсивных, которым отвеча- 
ют частоты $392(358), 414(426), 529(537), 581(586)$, 953 (1000), 1020 (1025), $1060(1074) \mathrm{cm}^{-1}$. В отличие от хлоратов интенсивности высокочастотных ИК-мод значительно больше интенсивностей рамановских мод. Так, для ИК-спектра $\mathrm{LiClO}_{4}\left(\mathrm{KClO}_{4}\right)$ в области высокочастотных внутримолекулярных колебаний выделяется область с тремя интенсивными максимумами при 969 (998), $1012(1020), \quad 1031(1052) \mathrm{cm}^{-1}$. Соответствующая область ИК-спектра перхлората натрия имеет только два максимума $\left(1015,1044 \mathrm{~cm}^{-1}\right)$. Что касается решеточных колебаний, то наиболее активными в ИК-спектре $\mathrm{NaClO}_{4}$ $\left(\mathrm{KClO}_{4}\right)$ являются моды с частотами $98(57), 142(81)$, $184(140) \mathrm{cm}^{-1}$, тогда как в ИК-спектре $\mathrm{LiClO}_{4}$ наиболее активны моды с частотами $125,163,254,306$, $361 \mathrm{~cm}^{-1}$. Области решеточных и внутримолекулярных колебаний перхлората лития практически перекрываются. Для $\mathrm{LiClO}_{4}\left(\mathrm{NaClO}_{4}, \mathrm{KClO}_{4}\right)$ решеточные моды с частотами $115(88,68), 161(150,114), 192(189,152) \mathrm{cm}^{-1}$ являются рамановскими, однако их интенсивности практически равны нулю. Интересно отметить, что частоты ИК- и рамановских мод решеточных колебаний хлоратов и перхлоратов с ростом радиуса катиона убывают.

На рис. 2 для $\mathrm{MgCa}\left(\mathrm{CO}_{3}\right)_{2}, \mathrm{NaClO}_{3}, \mathrm{KClO}_{3}$ и перхлоратов щелочных металлов представлены зависимости модовых параметров Грюнайзена $\gamma_{j}$ от частот $\omega_{j}$, рассчитанные при равновесном объеме. Частоты решеточных колебаний $\mathrm{MgCa}\left(\mathrm{CO}_{3}\right)_{2}, \mathrm{NaClO}_{3}, \mathrm{KClO}_{3}, \mathrm{LiClO}_{4}$, $\mathrm{NaClO}_{4}, \mathrm{KClO}_{4}$ не превышают 390, 240, 170, 380, 190, $150 \mathrm{~cm}^{-1}$ соответственно, и, таким образом, убывают с ростом атомной массы катиона и аниона. Наибольшие величины $\gamma_{j}$ для решеточных мод $\mathrm{MgCa}\left(\mathrm{CO}_{3}\right)_{2}, \mathrm{NaClO}_{3}$, $\mathrm{KClO}_{3}, \mathrm{LiClO}_{4}, \mathrm{NaClO}_{4}, \mathrm{KClO}_{4}$ составляют 2.3, 2.8, 3.9, $4.7,4.9,3.3$ соответственно, тогда как $\gamma_{j}$ для внутримолекулярных мод не превышают 0.4 (1.0 для $\left.\mathrm{LiClO}_{4}\right)$. Для $\mathrm{MgCa}\left(\mathrm{CO}_{3}\right)_{2}$ интересно отметить существование низкочастотных ИК-мод с существенно отрицательными параметрами Грюнайзена $-3.0,-1.6$. Также отрицательные величины параметра Грюнайзена низкочастотных мод были получены для кальцита $\left(\mathrm{CaCO}_{3}\right)$ в сравнительно недавней работе [55].

\section{4. Тепловые свойства}

В табл. 1 приведены коэффициенты зависимостей термодинамических потенциалов свободной $F_{T}$ и внутренней энергии $E_{T}$ от давления и температуры

$$
\begin{gathered}
f(P)=f_{0}+g_{1} P+g_{2} P^{2}, \\
f(T)=f_{0}+\sigma_{1}\left(T-T_{0}\right)+\sigma_{2}\left(T-T_{0}\right)^{2} .
\end{gathered}
$$

Здесь $T_{0}-$ стандартная температура, $f_{0}-$ значение величины при $P=0 \mathrm{GPa}$ и $T=T_{0}$, линейные коэффициенты $g_{1}$ и $\sigma_{1}$ показывают градиент, а коэффициенты $g_{2}$ и $\sigma_{2}$ характеризуют нелинейность. Максимальные величины $F_{T}$ и $E_{T}$ имеет доломит, а минимальные потенциалы соответствуют хлорату калия. При
Таблица 1. Коэффициенты $g_{i}, \sigma_{j}$ полиномиальной аппроксимации (16) термодинамических потенциалов свободной $F_{T}$ и внутренней $E_{T}$ энергии

\begin{tabular}{c|c|c|c|r|r|r}
\hline Кристалл & $\begin{array}{c}\text { Функция } f, \\
\mathrm{~kJ} \cdot \mathrm{mol}^{-1}\end{array}$ & $f_{0}$ & $g_{1}$ & $g_{2} \cdot 10^{2}$ & \multicolumn{1}{c|}{$\sigma_{1}$} & $\sigma_{2} \cdot 10^{3}$ \\
\hline $\mathrm{MgCa}\left(\mathrm{CO}_{3}\right)_{2}$ & $F_{T}(P, T)$ & 82.46 & 0.771 & -1.450 & -0.146 & -0.237 \\
& $E_{T}(P, T)$ & 127.0 & 0.368 & -0.244 & 0.155 & 0.284 \\
$\mathrm{NaClO}_{3}$ & $F_{T}(P, T)$ & 12.53 & 1.570 & -12.53 & -0.128 & -0.201 \\
& $E_{T}(P, T)$ & 49.87 & 0.254 & -0.991 & 0.095 & 0.127 \\
$\mathrm{KClO}_{3}$ & $F_{T}(P, T)$ & 5.139 & 2.454 & -7.731 & -0.154 & -0.234 \\
& $E_{T}(P, T)$ & 49.60 & 0.152 & 0.765 & 0.096 & 0.098 \\
$\mathrm{LiClO}_{4}$ & $F_{T}(P, T)$ & 26.46 & 2.347 & -16.74 & -0.131 & -0.204 \\
& $E_{T}(P, T)$ & 63.85 & 0.246 & 2.950 & 0.100 & 0.157 \\
$\mathrm{NaClO}_{4}$ & $F_{T}(P, T)$ & 16.49 & 3.161 & -31.12 & -0.169 & -0.251 \\
& $E_{T}(P, T)$ & 64.26 & 0.257 & 3.210 & 0.107 & 0.143 \\
$\mathrm{KClO}_{4}$ & $F_{T}(P, T)$ & 15.29 & 3.363 & -28.31 & -0.171 & -0.246 \\
& $E_{T}(P, T)$ & 63.74 & 0.205 & 0.960 & 0.106 & 0.138
\end{tabular}

Таблица 2. Параметры уравнения состояния кристаллов для ряда температур

\begin{tabular}{c|c|c|c|c|c}
\hline Кристалл & $T, \mathrm{~K}$ & 100 & 200 & 300 & 400 \\
\hline & $V_{0}, \AA^{3}$ & 107.453 & 107.566 & 107.759 & 107.967 \\
$\mathrm{MgCa}\left(\mathrm{CO}_{3}\right)_{2}$ & $B_{0}, \mathrm{GPa}$ & 107.82 & 106.68 & 105.36 & 104.14 \\
& $B_{1}$ & 3.18 & 3.19 & 3.21 & 3.21 \\
& $V_{0}, \AA^{3}$ & 72.21 & 72.721 & 73.381 & 74.066 \\
$\mathrm{NaClO}_{3}$ & $B_{0}, \mathrm{GPa}$ & 32.91 & 31.34 & 29.56 & 27.82 \\
& $B_{1}$ & 3.73 & 3.93 & 4.14 & 4.35 \\
& $V_{0}, \AA^{3}$ & 91.135 & 92.36 & 93.688 & 94.909 \\
$\mathrm{KClO}_{3}$ & $B_{0}, \mathrm{GPa}$ & 17.37 & 16.77 & 16.32 & 16.02 \\
& $B_{1}$ & 4.57 & 4.36 & 4.14 & 3.93 \\
& $V_{0}, \AA^{3}$ & 75.296 & 76.159 & 77.243 & 78.376 \\
$\mathrm{LiClO}_{4}$ & $B_{0}, \mathrm{GPa}$ & 27.96 & 25.78 & 23.50 & 21.40 \\
& $B_{1}$ & 5.54 & 5.78 & 6.00 & 6.19 \\
& $V_{0}, \AA^{3}$ & 84.782 & 85.914 & 87.39 & 88.981 \\
$\mathrm{NaClO}_{4}$ & $B_{0}, \mathrm{GPa}$ & 23.87 & 21.18 & 18.87 & 17.23 \\
& $B_{1}$ & 5.54 & 5.81 & 6.1 & 6.41 \\
& $V_{0}, \AA^{3}$ & 97.451 & 98.99 & 100.857 & 102.837 \\
$\mathrm{KClO}_{4}$ & $B_{0}, \mathrm{GPa}$ & 19.02 & 17.11 & 15.14 & 13.34 \\
& $B_{1}$ & 5.88 & 6.06 & 6.25 & 6.44
\end{tabular}

этом в изоанионных рядах с ростом атомного номера катиона $(\mathrm{Li} \rightarrow \mathrm{Na} \rightarrow \mathrm{K})$ потенциал $F_{T}$ уменьшается. Термодинамический потенциал $F_{T}$ убывает с ростом температуры и возрастает при повышении давления, тогда как внутренняя энергия $E_{T}$ возрастает в обоих случаях. Градиент $F_{T}$ по давлению максимален для перхлората калия $\left(3.36 \mathrm{~kJ} \cdot \mathrm{mol}^{-1} \cdot \mathrm{GPa}^{-1}\right)$ и минимален для доломита $\left(0.77 \mathrm{~kJ} \cdot \mathrm{mol}^{-1} \cdot \mathrm{GPa}^{-1}\right)$. Скорость убывания $F_{T}$ с температурой минимальна для хлората натрия, перхлората лития и с ростом атомной массы катиона 

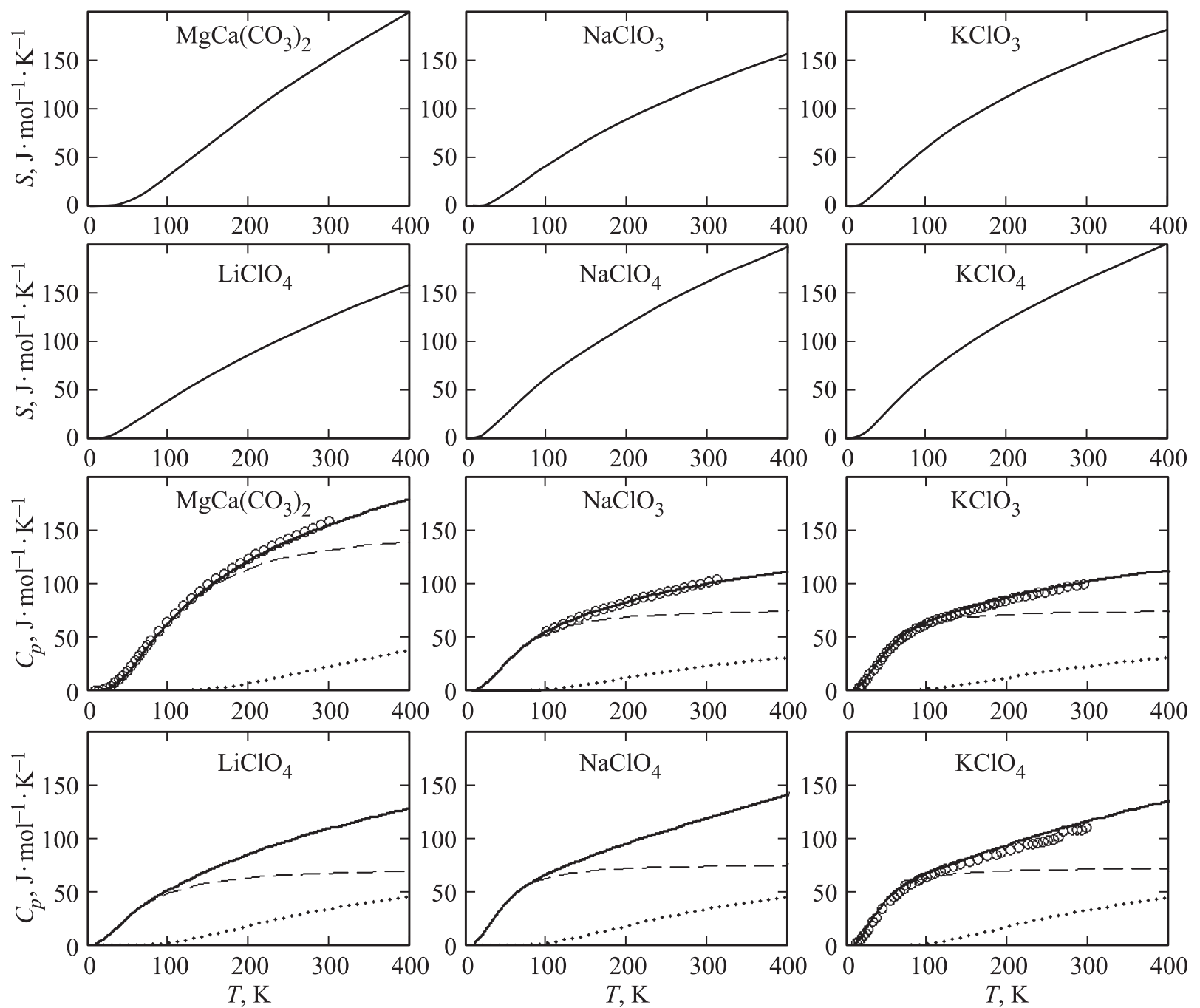

Рис. 3. Температурные зависимости теплоемкости $C_{p}$ и энтропии $S$, рассчитанные в настоящей работе (сплошная линия), экспериментальные данные [25-28] (кружки), а также вклады решеточных колебаний (штриховые линии) и внутримолекулярных колебаний (пунктирные линии) в теплоемкость $C_{V}$ для оксианионных кристаллов.

увеличивается (табл. 1). Что касается $E_{T}$, то скорость ее изменения с давлением максимальна для перхлората натрия $\left(0.26 \mathrm{~kJ} \cdot \mathrm{mol}^{-1} \cdot \mathrm{GPa}^{-1}\right)$ и минимальна для хлората калия $\left(0.15 \mathrm{~kJ} \cdot \mathrm{mol}^{-1} \cdot \mathrm{GPa}^{-1}\right)$. Наименьший градиент $E_{T}$ по температуре соответствует доломиту и хлорату натрия.

В табл. 2 приведены вычисленные параметры уравнения состояния (12) для доломита, хлоратов и перхлоратов щелочных металлов. Данные табл. 2 позволяют определить аналитическую зависимость $P(V, T)$ для изотерм $T=100,200,300,400 \mathrm{~K}$. Из таблицы видно, что в изоанионных рядах равновесные значения объема $V_{0}$ (для формульной единицы) при увеличении атомного радиуса катиона увеличиваются, тогда как объемные модули $B_{0}$ существенно уменьшаются. Объемы $V_{0}$ для перхлоратов и доломита больше, чем для хлоратов. Объемный модуль $B_{0}$ для доломита максимален. Кроме того, небольшие изменения происходят с ростом температуры. Так, при увеличении температуры от 100 до $300 \mathrm{~K}$, равновесный объем хлората калия увеличивается на 2.8\% (экспериментальное значение 2.1\% [13]). Для перхлората калия это изменение объема составляет $3.5 \%(2.7 \%[16,56])$, тогда как для доломита всего $0.3 \%$. Существенно уменьшается при нагревании и значение $B_{0}$, в особенности для перхлоратов. Так, при увеличении температуры от 100 до $300 \mathrm{~K}$ объемный модуль перхлората калия уменьшается на 20.4\%. Отметим, что экспериментальные значения объемных модулей составляют 94.0, 27.9, 19.2, 24.3, 24.1, 21.3 GPa [57,58], и удовлетворительно согласуются с расчетными. Здесь и далее численные значения приводятся в последовательности: $\mathrm{MgCa}\left(\mathrm{CO}_{3}\right)_{2}, \mathrm{NaClO}_{3}, \mathrm{KClO}_{3}, \mathrm{LiClO}_{4}, \mathrm{NaClO}_{4}$, $\mathrm{KClO}_{4}$. Коэффициенты Пуассона $\mu$ согласно [37,59] равны $0.269,0.276,0.309,0.278,0.297,0.319$. Таким образом, согласно (14) температура Дебая для рассматриваемых оксианионных кристаллов составляет 675, 344, 227, 308, 265,215 K. Наибольшая температура Дебая отвечает доломиту, хлорату натрия, перхлорату лития и в изо- 
Таблица 3. Теплоемкость при постоянном объеме $C_{V}\left(\mathrm{~J} \cdot \mathrm{mol}^{-1} \cdot \mathrm{K}^{-1}\right)$, параметр Грюнайзена $\gamma$, коэффициент объемного расширения $\alpha\left(10^{-4} \mathrm{~K}^{-1}\right)$ для ряда температур и давлений $0,1 \mathrm{GPa}$ (в скобках)

\begin{tabular}{|c|c|c|c|c|c|c|c|}
\hline \multirow[b]{2}{*}{$T, \mathrm{~K}$} & \multirow[b]{2}{*}{ Параметр } & \multicolumn{6}{|c|}{ Оксианионные кристаллы } \\
\hline & & $\begin{array}{l}\mathrm{MgCa} \\
\left(\mathrm{CO}_{3}\right)_{2}\end{array}$ & $\mathrm{NaClO}_{3}$ & $\mathrm{KClO}_{3}$ & $\mathrm{LiClO}_{4}$ & $\mathrm{NaClO}_{4}$ & $\mathrm{KClO}_{4}$ \\
\hline \multirow{3}{*}{100} & $C_{V}$ & $\begin{array}{c}62.03 \\
(61.17)\end{array}$ & $\begin{array}{c}54.37 \\
(52.47)\end{array}$ & $\begin{array}{c}63.07 \\
(60.78)\end{array}$ & $\begin{array}{c}49.82 \\
(47.88)\end{array}$ & $\begin{array}{c}64.62 \\
(62.16)\end{array}$ & $\begin{array}{c}65.32 \\
(63.08)\end{array}$ \\
\hline & $\gamma$ & $\begin{array}{c}0.53 \\
(0.46)\end{array}$ & $\begin{array}{c}1.40 \\
(1.27)\end{array}$ & $\begin{array}{c}1.83 \\
(1.88)\end{array}$ & $\begin{array}{c}2.35 \\
(2.27)\end{array}$ & $\begin{array}{c}1.97 \\
(1.45)\end{array}$ & $\begin{array}{c}2.34 \\
(2.18)\end{array}$ \\
\hline & $\alpha$ & $\begin{array}{c}0.05 \\
(0.04)\end{array}$ & $\begin{array}{c}0.53 \\
(0.43)\end{array}$ & $\begin{array}{c}1.22 \\
(1.00)\end{array}$ & $\begin{array}{c}0.92 \\
(0.74)\end{array}$ & $\begin{array}{c}1.05 \\
(0.63)\end{array}$ & $\begin{array}{c}1.39 \\
(1.00)\end{array}$ \\
\hline \multirow{3}{*}{200} & $C_{V}$ & $\begin{array}{c}120.79 \\
(119.92)\end{array}$ & $\begin{array}{c}80.71 \\
(79.71)\end{array}$ & $\begin{array}{c}83.67 \\
(82.83)\end{array}$ & $\begin{array}{c}80.62 \\
(79.44)\end{array}$ & $\begin{array}{c}89.83 \\
(88.79)\end{array}$ & $\begin{array}{c}88.37 \\
(87.54)\end{array}$ \\
\hline & $\gamma$ & $\begin{array}{c}0.87 \\
(0.83)\end{array}$ & $\begin{array}{c}1.40 \\
(1.28)\end{array}$ & $\begin{array}{c}1.57 \\
(1.64)\end{array}$ & $\begin{array}{c}1.87 \\
(1.80)\end{array}$ & $\begin{array}{c}1.83 \\
(1.44)\end{array}$ & $\begin{array}{c}1.99 \\
(1.86)\end{array}$ \\
\hline & $\alpha$ & $\begin{array}{c}0.15 \\
(0.14)\end{array}$ & $\begin{array}{c}0.83 \\
(0.68)\end{array}$ & $\begin{array}{c}1.41 \\
(1.23)\end{array}$ & $\begin{array}{c}1.27 \\
(1.02)\end{array}$ & $\begin{array}{c}1.52 \\
(0.98)\end{array}$ & $\begin{array}{c}1.74 \\
(1.26)\end{array}$ \\
\hline \multirow{3}{*}{300} & $C_{V}$ & $\begin{array}{c}154.25 \\
(153.64)\end{array}$ & $\begin{array}{c}95.36 \\
(94.75)\end{array}$ & $\begin{array}{c}96.56 \\
(96.13)\end{array}$ & $\begin{array}{c}100.73 \\
(100.02)\end{array}$ & $\begin{array}{c}107.42 \\
(106.78)\end{array}$ & $\begin{array}{c}105.46 \\
(104.97)\end{array}$ \\
\hline & $\gamma$ & $\begin{array}{c}0.89 \\
(0.86)\end{array}$ & $\begin{array}{c}1.33 \\
(1.21)\end{array}$ & $\begin{array}{c}1.37 \\
(1.46)\end{array}$ & $\begin{array}{c}1.62 \\
(1.56)\end{array}$ & $\begin{array}{c}1.71 \\
(1.37)\end{array}$ & $\begin{array}{c}1.75 \\
(1.64) \\
\end{array}$ \\
\hline & $\alpha$ & $\begin{array}{c}0.20 \\
(0.19)\end{array}$ & $\begin{array}{c}0.98 \\
(0.80)\end{array}$ & $\begin{array}{c}1.44 \\
(1.30)\end{array}$ & $\begin{array}{c}1.53 \\
(1.17)\end{array}$ & $\begin{array}{c}1.96 \\
(1.25)\end{array}$ & $\begin{array}{c}2.02 \\
(1.43)\end{array}$ \\
\hline \multirow{3}{*}{400} & $C_{V}$ & $\begin{array}{c}177.35 \\
(176.89) \\
\end{array}$ & $\begin{array}{c}104.58 \\
(104.16) \\
\end{array}$ & $\begin{array}{c}105.11 \\
(104.85)\end{array}$ & $\begin{array}{c}113.97 \\
(113.52) \\
\end{array}$ & $\begin{array}{c}119.62 \\
(119.16) \\
\end{array}$ & $\begin{array}{c}117.46 \\
(117.14)\end{array}$ \\
\hline & $\gamma$ & $\begin{array}{c}0.87 \\
(0.84)\end{array}$ & $\begin{array}{c}1.3 \\
(1.17)\end{array}$ & $\begin{array}{c}1.24 \\
(1.34)\end{array}$ & $\begin{array}{c}1.51 \\
(1.43)\end{array}$ & $\begin{array}{c}1.68 \\
(1.36)\end{array}$ & $\begin{array}{c}1.63 \\
(1.52)\end{array}$ \\
\hline & $\alpha$ & $\begin{array}{c}0.23 \\
(0.22)\end{array}$ & $\begin{array}{c}1.11 \\
(0.89)\end{array}$ & $\begin{array}{c}1.42 \\
(1.32)\end{array}$ & $\begin{array}{l}1.937 \\
(1.29)\end{array}$ & $\begin{array}{c}2.59 \\
(1.55)\end{array}$ & $\begin{array}{c}2.37 \\
(1.61)\end{array}$ \\
\hline
\end{tabular}

анионных рядах с ростом атомного номера катиона она уменьшается.

На рис. 3 для доломита, хлоратов и перхлоратов щелочных металлов представлены вычисленные температурные зависимости энтропии $S$ и теплоемкости $C_{p}$ (сплошные линии), а также экспериментальные данные [25-28] (кружки). Также на рис. 3 приведены вклады решеточных колебаний (штриховые линии) и внутримолекулярных колебаний (пунктирные линии) в теплоемкость $C_{V}$. Видно, что рассчитанные зависимости хорошо согласуются с экспериментальными.

Энтропия монотонно возрастает с ростом температуры и при стандартной температуре достигает значений $149,125,149,125,160,162 \mathrm{~J} \cdot \mathrm{mol}^{-1} \cdot \mathrm{K}^{-1}$. Таким образом, в изокатионных рядах энтропия хлоратов меньше, чем перхлоратов, а при увеличении радиуса катиона энтропия увеличивается. По величинам стандартных молярных энтропий химических соединений можно судить об особенностях их внутренней струк- туры. Поскольку энтропия хлората натрия и перхлората лития является наименьшей, то эти соединения имеют наиболее упорядоченную структуру. Хлораты натрия и калия более упорядочены, чем соответствующие перхлораты.

Теплоемкость $C_{p}$ при температурах больших $\sim 100-200 \mathrm{~K}$ монотонно возрастает практически по линейному закону (рис. 3). Рассчитанные значения молярной теплоемкости $C_{p}$ при стандартной температуре равны $155(158), 99(100), 102(101), 108,118$, $117(110) \mathrm{J} \cdot \mathrm{mol}^{-1} \cdot \mathrm{K}^{-1}$. В скобках приведены экспериментальные данные [25-28]. Таким образом, наибольшая теплоемкость отвечает доломиту, а теплоемкость хлоратов меньше, чем перхлоратов. Из рис. 3 видно, что решеточные колебания практически полностью определяют поведение теплоемкости при низких температурах (меньших $100 \mathrm{~K}$ ). С повышением температуры вклады решеточных колебаний стремятся к постоянной величине и температурная зависимость теплоемкости определяется вкладами внутримолекулярных колебаний, 
которые при температурах больших $\sim 100-200 \mathrm{~K}$ почти линейно возрастают.

В табл. 3 приведены теплоемкость при постоянном объеме $C_{V}$, параметр Грюнайзена $\gamma$ и коэффициент объемного расширения $\alpha$ оксианионных кристаллов, вычисленные для ряда температур при давлениях 0 и $1 \mathrm{GPa}$. Для температуры $100 \mathrm{~K}$ теплоемкость $C_{V}$ при увеличении массы катиона возрастает (табл. 3). Кроме того, теплоемкость $C_{V}$ возрастает с ростом температуры. Для каждой температуры $C_{V}$ уменьшается с ростом $P$, причем для высоких температур и масс катиона менее значимо. Так, при температуре $200 \mathrm{~K}$ увеличение давления до $1 \mathrm{GPa}$ вызывает уменьшение теплоемкости перхлората лития (хлората калия) на $1.18(0.84) \mathrm{J} \cdot \mathrm{mol}^{-1} \cdot \mathrm{K}^{-1}$, тогда для температуры $400 \mathrm{~K}$ соответствующая величина составляет $0.45(0.26) \mathrm{J} \cdot \mathrm{mol}^{-1} \cdot \mathrm{K}^{-1}$. Наименьшее значение параметр Грюнайзена имеет для доломита, тогда как максимален для $\mathrm{LiClO}_{4}$ (при $100 \mathrm{~K}$ ), $\mathrm{KClO}_{4}$ (при $300 \mathrm{~K}$ ). $\mathrm{C}$ ростом температуры параметр Грюнайзена для хлоратов и перхлоратов уменьшается, а для доломита $\gamma$ увеличивается. При $T=300 \mathrm{~K}$ параметр в изоанионных рядах $\mathrm{Li} \rightarrow \mathrm{Na} \rightarrow \mathrm{K}$ увеличивается. Для $\mathrm{NaClO}_{3}, \mathrm{NaClO}_{4}$, $\mathrm{KClO}_{4}$ при стандартных условиях экспериментальные значения $\gamma$ равны $1.37,1.56,1.64$ [60] соответственно и хорошо согласуются с вычисленными (табл. 3). В отличие от других оксианионных кристаллов, параметр Грюнайзена хлората калия под давлением увеличивается. Что касается коэффициента объемного расширения, то вычисленный $\alpha$ является минимальным для доломита и максимальным для перхлората калия. Для доломита, хлората натрия, хлората и перхлората калия при стандартных условиях экспериментальные значения коэффициента $\alpha$ равны $0.26 \cdot 10^{-4} \mathrm{~K}^{-1}[29], 1.21 \cdot 10^{-4} \mathrm{~K}^{-1}$ [30], $1.34 \cdot 10^{-4} \mathrm{~K}^{-1}$ [31] и $1.53 \cdot 10^{-4} \mathrm{~K}^{-1}$ [32] соответственно. С ростом температуры и массы катиона $\alpha$ увеличивается. Исключение составляет хлорат калия, для которого коэффициент объемного расширения при температуре $400 \mathrm{~K}$ меньше, чем при $T=300 \mathrm{~K}$ (табл. 3). Это согласуется с экспериментальными измерениями [31]. Под давлением коэффициент объемного расширения уменьшается. Наибольший эффект давление оказывает на коэффициент $\alpha$ перхлората натрия, тогда как наименьший отвечает доломиту. В отличие от $C_{V}$, зависимость $\alpha$ от давления для высоких температур более значительна, чем для низких (за исключением $\mathrm{KClO}_{3}$ ). Например, коэффициент $\alpha$ хлората натрия при температуре $100 \mathrm{~K}$ под давлением уменьшается как $0.10 \cdot 10^{-4} \mathrm{~K}^{-1} / \mathrm{GPa}$, а при $400 \mathrm{~K}$ как $0.22 \cdot 10^{-4} \mathrm{~K}^{-1} / \mathrm{GPa}$. При стандартных условиях коэффициенты линейного расширения $\alpha_{l}=-(\partial l / \partial T) / l$, вычисленные вдоль кристаллографических осей $a, b, c$, имеют следующие значения (в скобках приведены экспериментальные данные [29-32]): $\alpha_{a}=0.04(0.04)$, $0.33(0.40), 0.37(0.55), 0.18,0.69,0.98(0.71) \cdot 10^{-4} \mathrm{~K}^{-1}$; $\alpha_{b}=0.04(0.04), \quad 0.33(0.40), \quad 0.33(0.39), \quad 0.78, \quad 0.62$, $0.38(0.39) \cdot 10^{-4} \mathrm{~K}^{-1} ; \quad \alpha_{c}=0.12(0.18), \quad 0.33(0.40)$, $0.83(0.74), \quad 0.56, \quad 0.65, \quad 0.66(0.43) \cdot 10^{-4} \mathrm{~K}^{-1}$. Таким образом, тепловое расширение доломита, хлората калия, перхлората лития и калия существенно анизотропно.

\section{5. Заключение}

Колебательные и тепловые свойства оксианионных кристаллов изучены на основе теории функционала плотности и квазигармонической модели ДебаяЭйнштейна. Вычисленные величины находятся в хорошем согласии с имеющимися экспериментальными данными.

В ИК- и КР-спектрах выделяются полосы, отвечающие решеточным колебаниям, и интенсивные пики высокочастотных внутримолекулярных колебаний. Внутримолекулярные моды, активные в ИК и КР-спектрах хлоратов и перхлоратов, имеют меньшие частоты, чем высокочастотные моды доломита. Если в рамановских спектрах доломита и хлоратов выделяется три области активных внутримолекулярных колебаний, то в рамановских спектрах перхлоратов их четыре и пики при $\sim 850 \mathrm{~cm}^{-1}$ самые интенсивные. При этом для $\mathrm{KClO}_{4}$ эти области более узкие, чем для перхлоратов лития и натрия. В отличие от хлоратов интенсивности высокочастотных ИК-мод перхлоратов значительно больше интенсивностей рамановских мод. Частоты мод решеточных колебаний убывают с ростом атомной массы катиона и аниона, а параметры Грюнайзена для большинства этих мод значительно больше, чем для внутримолекулярных. Для доломита имеются низкочастотные ИК-моды с существенно отрицательными параметрами Грюнайзена.

Максимальные величины свободной и внутренней энергии имеет доломит, а минимальные соответствуют хлорату калия. При этом градиент свободной энергии по давлению максимален для перхлората калия и минимален для доломита. Скорость изменения внутренней энергии с давлением максимальна для перхлората натрия и минимальна для хлората калия.

Сушественно уменьшается при нагревании значение объемного модуля, в особенности для перхлоратов. Наибольшая температура Дебая отвечает доломиту, хлорату натрия, перхлорату лития и в изоанионных рядах с ростом атомного номера катиона она уменьшается.

В изокатионных рядах энтропия хлоратов меньше, чем перхлоратов, а при увеличении радиуса катиона она увеличивается. Наибольшая теплоемкость отвечает доломиту, а теплоемкость хлоратов меньше, чем перхлоратов. Решеточные колебания практически полностью определяют поведение теплоемкости при температурах меньших $100 \mathrm{~K}$, тогда как внутримолекулярные колебания преимущественно определяют поведение теплоемкости при температурах больших $200 \mathrm{~K}$.

Для температуры $100 \mathrm{~K}$ теплоемкость $C_{V}$ при увеличении массы катиона возрастает. Для каждой температуры $C_{V}$ уменьшается с ростом давления, причем для высоких температур и масс катиона менее значимо. Наименьшее значение параметр Грюнайзена имеет 
для доломита, тогда как максимален для перхлоратов. С ростом температуры параметр Грюнайзена для хлоратов и перхлоратов уменьшается, а для доломита увеличивается. В отличие от других оксианионных кристаллов, параметр Грюнайзена хлората калия под давлением увеличивается. Коэффициент объемного расширения $\alpha$ является минимальным для доломита, максимальным для перхлората калия и с ростом температуры увеличивается. Исключение составляет хлорат калия, для которого коэффициент $\alpha$ при температуре $400 \mathrm{~K}$ меньше, чем при $300 \mathrm{~K}$. Наибольший эффект давление оказывает на коэффициент $\alpha$ перхлората натрия, тогда как наименьший отвечает доломиту. В отличии от $C_{V}$, зависимость $\alpha$ от давления для высоких температур более значительна, чем для низких (за исключением $\mathrm{KClO}_{3}$ ). Тепловое расширение доломита, хлората калия, перхлората лития и калия существенно анизотропно.

\section{Список литературы}

[1] M. Javoy, F. Pineau, C. Allbgre. Nature 300, 171 (1982).

[2] Y. Zhang, A. Zindler. Earth. Earth Planet. Sci. Lett. 117, 331 (1993).

[3] N.V. Solomatova, P.D. Asimow. Am. Mineral. 102, 210 (2017).

[4] A. Radenovic. Kem. Ind. 55, 65 (2006).

[5] M. Samtami, D. Dollimore, F. Wilburn, K. Alexander. Thermochim. Acta 367, 285 (2001).

[6] G.P. Sutton, O. Biblarz. Rocket Propulsion Elements. John Wiley Sons Inc., New Jersey (2010).

[7] G. Steinhauser, T.M. Klapotke. Angew. Chem. Int. Ed. 47, 3330 (2008).

[8] E.C. Koch. Prop. Exp. Pyrotech. 29, 67 (2004).

[9] S.U. Patil, S.S. Yawale, S.P. Yawale. Bull. Mater. Sci. 37, 1403 (2014).

[10] M.H. Hecht, S.P. Kounaves, R.C. Qurinn, S.J. West, S.M. Young, D.W. Ming, D.C. Catling, B.C. Clark, W.V. Boynton, J. Hoffman, L.P. DeFlores, K. Gospodinova, J. Kapit, P.H. Smith. Science 325, 64 (2009).

[11] H. Effenberger, K. Mereiter, J. Zemann. Z. Krist. 156, 233 (1981).

[12] S.C. Abrahams, J.L. Bernstein. Acta. Cryst. B 33, 3601 (1977).

[13] J. Danielsen, A. Hazel, F. Larsen. Acta. Cryst. B 37, 913 (1981).

[14] M.S. Wickleder. Z. Anorg. Allg. Chem. 629, 1466 (2003).

[15] R. Wartchow, H.J. Berthold. Z. Krist. 147, 307 (1978).

[16] J.W. Bats, H. Fuess. Acta. Cryst. B 38, 2116 (1982).

[17] P. Gillet, C. Biellmann, B. Reynard, P. McMillan. Phys. Chem. Minerals 20, 1 (1993).

[18] S. Gunasekaran, G. Anbalagan, S. Pandi. J. Raman Spectrosc. 37, 892 (2006).

[19] J. Sun, Z. Wua, H. Cheng, Z. Zhang, R.L. Frost. Spectrochim. Acta A 117, 158 (2014).

[20] G. Andermann, D.A. Dows. J. Phys. Chem. Solids 28, 1307 (1967).

[21] J. Bates. J. Chem. Phys. 55, 494 (1971).

[22] Y.H. Zhang, C.K. Chan. J. Phys. Chem. A 107, 5956 (2003).

[23] H. Lutz, R. Becker. Spectrochim. Acta A 35, 797 (1979).

[24] M. Pravica, Y. Wang, D. Sneed, S. Reiser, M. White. Chem. Phys. Lett. 660, 37 (2016).
[25] J.W. Zhang, R.A. Robie. J. Phys. Chem. 67, 2248 (1963).

[26] A. Fransson, R.G. Ross. J. Phys. C 16, 2861 (1983).

[27] W. Latimer, P. Schutz, J. Hicks. J. Am. Chem. Soc. 56, 88 (1934).

[28] W. Latimer, J. Ahlberg. J. Am. Chem. Soc. 52, 549 (1930).

[29] R. Reeder, S. Markgraf. Am. Mineral. 71, 795 (1986).

[30] S. Ganesan. J. Ind. Inst. Sci. 41, 9 (1959).

[31] M.A. Lonappan. Proc. Phys. Soc. B 68, 75 (1955).

[32] S. Haussuhl. Z. Krist. 192, 137 (1990).

[33] L. Valenzano, Y. Noel, R. Orlando, C. Zicovich-Wilson, M. Ferrero, R. Dovesi. Theor. Chem. Acc. 117, 991 (2007).

[34] Y. Yedukondalu, G. Vaitheeswaran. Mater. Chem. Phys. 181, 54 (2016).

[35] Д.В. Корабельников, Ю.Н. Журавлёв. Поверхность. Рентген., синхротр. и нейтрон. исслед. 11, 55 (2013).

[36] Ю.Н. Журавлёв, Д.В. Корабельников. Изв. вузов. Физика 9, 70 (2009).

[37] Д.В. Корабельников, Ю.Н. Журавлев. ФТТ 58, 1129 (2016).

[38] Д.В. Корабельников, Ю.Н. Журавлев. ФТТ 59, 248 (2017).

[39] R. Dovesi, R. Orlando, A. Erba, C.M. Zicovich-Wilson, B. Civalleri, S. Casassa, L. Maschio, M. Ferrabone, M. De La Pierre, Ph. D’Arco. Int. J. Quantum Chem. 114, 1287 (2014).

[40] M. Catti, R. Dovesi, A. Pavese, V. Saunders. J. Phys. Condens. Matter. 3, 4151 (1991).

[41] L. Ojamae, K. Hermansson, C. Pisani, M. Causa, C. Roetti. Acta Cryst. B 50, 268 (1994).

[42] R. Dovesi, C. Roetti, C. Fava, M. Prencipe, V.R. Saunders. Chem. Phys. 156, 11 (1991).

[43] M. Catti, A. Pavese, R. Dovesi, V. Saunders. Phys. Rev. B 47, 9189 (1993).

[44] C. Gatti, V. Saunders, C. Roetti. J. Chem. Phys. 101, 10686 (1994).

[45] M.S. Gordon, J.S. Binkley, J.A. Pople, W.J. Pietro, W.J. Hehre. J. Am. Chem. Soc. 104, 2797 (1982).

[46] J.P. Perdew, Y. Wang. Phys. Rev. B 45, 13244 (1992).

[47] C.G. Broyden. J. Appl. Math. 6, 222 (1970).

[48] J. Maul, A. Erba, I. Santos, J. Sambrano, R. Dovesi. J. Chem. Phys. 142, 014505 (2015).

[49] L. Maschio, B. Kirtman, R. Orlando, M. Rerat. J. Chem. Phys. 137, 204113 (2012)

[50] L. Maschio, B. Kirtman, M. Rerat, R. Orlando, R. Dovesi. J. Chem. Phys. 139, 164102 (2013).

[51] P. Vinet, J.H. Rose, J. Ferrante, J.R. Smith. J. Phys.: Condens Matter 1, 1941 (1989).

[52] S. Baroni, P. Giannozzi, E. Isaev. Rev. Mineral. \& Geochem. 71, 39 (2010).

[53] A. Otero-de-la-Rosa, D. Abbasi-Perez, V. Luana. Comp. Phys. Commun. 182, 2232 (2011).

[54] E. Francisco, J.M. Recio, M.A. Blanco, A.M. Pendas, A. Costales. J. Phys. Chem. A 102, 1595 (1998).

[55] C.G. Ungureanu, R. Cossio, M. Prencipe. CALPHAD 37, 25 (2012).

[56] D. Marabello, R. Bianchi, G. Gervasio, F. Cargnoni. Acta Cryst. A 60, 494 (2004).

[57] N.L. Ross, R.J. Reeder. Am. Mineral. 77, 412 (1992).

[58] P.W. Bridgman. Proc. Am. Acad. Art. Sci. 76, 9 (1945).

[59] P.-F. Chen, L.-Y. Chiao, P.-H. Huang. Phys. Earth Planet. Interiors 155, 73 (2006).

[60] В.Н. Беломестных, Е.П. Теслева. ЖТФ 74, 8, 140 (2004). 